\title{
Chewing gum as a non-pharmacological alternative for orthodontic pain relief: A randomized clinical trial using an intention-to-treat analysis
}

Diego Junior da

Silva Santos ${ }^{\mathrm{a}, \mathrm{b}}$ (1)

Jonas Capelli Jr. ${ }^{\mathrm{a}}$ (1)

${ }^{a}$ Department of Preventive and Community Dentistry, School of Dentistry, Rio de Janeiro State University, Rio de Janeiro, Brazil

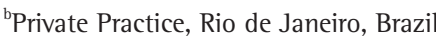

Objective: To compare the effectiveness of ibuprofen, acetaminophen, and chewing gum for orthodontic pain relief and to assess if chewing gum can be a non-pharmacological alternative for orthodontic pain relief. Methods: The study enrolled 106 patients of both sexes, aged $\geq 12$ years, with body weight $>50 \mathrm{~kg}$, and mild-to-moderate dental crowding in the upper arch. After randomization and allocation concealment, the intervention groups were either administered with ibuprofen $(400 \mathrm{mg})$ or acetaminophen $(500 \mathrm{mg})$ or chewed sugar-free chewing gum immediately after initial archwire placement and every 6 hours for 1 week if the pain persisted. The control group did not receive any pain relief. The pain was assessed on a $100-\mathrm{mm}$ visual analog scale at rest and while biting down at T1 (2 hours), T2 (24 hours), T3 (2 days), T4 (3 days), T5 (7 days), and T6 (21 days). Statistical analyses were performed using the Kruskal-Wallis and post-hoc Mann-Whitney $U$ tests $(\alpha=0.05)$. Results: The chewing gum group experienced more pain relief than the ibuprofen group at while biting down at T3 $(p=0.04)$ and at rest at T4 $(p<0.001)$. The chewing gum group reported more pain relief than the acetaminophen and control groups while biting down at T3 $(p=0.03$ and $p=0.0006$, respectively) and T4 (both $p<0.001)$. Conclusions: Chewing gum can be a non-pharmacological alternative for orthodontic pain relief at 2 and 3 days after initial archwire placement.

[Korean J Orthod 2021;51(5):346-354]

Key words: Pain, lbuprofen, Acetaminophen, Randomized clinical trial

Received August 14, 2020; Revised January 4, 2021; Accepted February 1, 2021.

Corresponding author: Jonas Capelli Jr.

Professor, Department of Preventive and Community Dentistry, School of Dentistry, Rio de Janeiro State University, Boulevard 28 de setembro, 157, 2nd floor, Vila lsabel, Rio de Janeiro-RJ 20551-030, Brazil.

Tel +55-21-2569-3829 e-mail jonascapelli@gmail.com

How to cite this article: da Silva Santos DJ, Capelli J Jr. Chewing gum as a nonpharmacological alternative for orthodontic pain relief: A randomized clinical trial using an intention-to-treat analysis. Korean J Orthod 2021;51:346-354. (C) 2021 The Korean Association of Orthodontists.

This is an Open Access article distributed under the terms of the Creative Commons Attribution Non-Commercial License (http://creativecommons.org/licenses/by-nc/4.0) which permits unrestricted non-commercial use, distribution, and reproduction in any medium, provided the original work is properly cited. 


\section{INTRODUCTION}

Medical treatment, especially using nonsteroidal antiinflammatory drugs (NSAIDs) and acetaminophen, is considered the most successful modality for reducing orthodontic pain. ${ }^{1,2}$ However, several reports have suggested non-pharmacological methods for orthodontic pain relief, including low-level laser therapy ${ }^{3,4}$ and masticatory stimulation of the periodontal ligament. ${ }^{5}$ These alternatives also help overcome the side effects of NSAIDs and acetaminophen, such as stomach problems, allergic reactions, increased blood pressure, and liver toxicity. ${ }^{6}$ In this context, patients and orthodontists have pursued a non-pharmacological and widely available method for orthodontic pain relief that has no negative side effects but some positive ones, i.e., stimulating the production of saliva. $^{\text {? }}$

The chewing of gum or use of bite wafers has been reported to alleviate pain induced by the application of orthodontic force. Chewing rubbery materials allows blood flow in compressed areas of the periodontal ligament and prevents the formation of pain-inducing inflammatory metabolites, thereby attenuating pain perception. ${ }^{3}$ Furthermore, chewing gum is widely available at a low cost.

Several studies have reported the use of bite wafers as a non-pharmacological option for orthodontic pain relief..$^{8-10}$ The use of bite wafers like chewing gum loosens the tightly grouped fibers around the nerves and blood vessels, restores normal vascular and lymphatic circulation, and prevents or relieves inflammation in the periodontal ligament. ${ }^{11}$

Other reports compared the effectiveness of chewing gum and ibuprofen for orthodontic pain relief and confirmed that chewing gum and bite wafers could be recommended as substitutes to ibuprofen., ${ }^{5,6}$ In addition, studies have shown that chewing gum does not increase the rate of appliance breakages. ${ }^{12,13}$

Other non-pharmacological methods for orthodontic pain relief have also been reported, such as laser therapy and verbal behavior modification. Bayani et al. ${ }^{3}$ asserted that lasers emitting infrared radiation could be a substitute to ibuprofen and could relief pain comparable to bite wafers. A recent evaluation of the effectiveness of verbal behavior modification and acetaminophen on orthodontic pain demonstrated that a psychological approach can be an adjunct to analgesics for patients receiving orthodontic treatments. However, psychological management alone is ineffective in managing orthodontic pain. ${ }^{14}$

Several studies have compared the effectiveness of pharmacological methods for orthodontic pain. ${ }^{5,6,15}$ However, no study to date has compared the two most prescribed drugs in orthodontics (ibuprofen and acet- aminophen) with chewing gum in terms of achieving orthodontic pain relief. Moreover, in a systematic review, Fleming et al. ${ }^{16}$ reported very low-quality evidence and inconsistent data regarding the relief of self-reported pain associated with the use of chewing adjuncts.

Against this background, the current study aimed to compare the effectiveness of chewing gum, ibuprofen, and acetaminophen on pain relief after initial archwire placement in patients receiving orthodontic treatment. The null hypothesis was that there will be no significant differences in pain relief among patients receiving ibuprofen, acetaminophen, and chewing gum. To date, few randomized controlled trials (RCTs) have been included in systematic reviews on the methods for managing orthodontic pain. Moreover, to the best of our knowledge, no RCTs have compared chewing gum to ibuprofen and acetaminophen for pain relief in orthodontic treatment.

\section{MATERIALS AND METHODS}

\section{Ethical aspects}

The Ethics Committee of Rio de Janeiro State University approved this study (CAAE: 01583112.3.0000.5259), and all patients provided written informed consent. This study was registered with and can be accessed at ClinicalTrials.gov (ID: NCT03568721).

\section{Study design}

This study was a parallel, single-blind, single-center RCT that was conducted in Rio de Janeiro, Brazil, between January 2015 and March 2018.

\section{Patients}

Patients who required orthodontic treatment were consecutively selected by residents in the Residency Program in Orthodontics, Department of Preventive and Community Dentistry, Rio de Janeiro State University, Rio de Janeiro, Brazil. The eligibility criteria were body weight $>50 \mathrm{~kg}$, mild-to-moderate dental crowding in the upper arch allowing the insertion of an initial 0.014" nickel-titanium archwire, and no previous history of orthodontic treatment. Patients aged $<12$ years old; those with active periodontal disease, allergies, contraindication for ibuprofen and acetaminophen, or autoimmune diseases; and those who were pregnant, or lactating were excluded.

\section{Randomization}

Allocation concealment consisted of randomization that was performed by the first author (DJSS) in 12 blocks, with a block size of 12, by using a computergenerated (www.randomization.com) random number table with an allocation ratio of 1:1:1:1. lbuprofen and 
acetaminophen were administered as capsules, which were identical in appearance. They were prepacked in bottles and labeled by a pharmacy company as follows: FORMULA 1 (ibuprofen) and FORMULA 2 (acetaminophen). The legend was kept secret by the pharmacy company until the end of the trial. The allocation sequence was also concealed from the authors (DJSS and $\mathrm{JCJ}$ ), with enrollment and assessment based on sequentially numbered, opaque, sealed, and stapled envelopes. A departmental assistant handled the envelopes and assigned the patients to various interventions. Although the chewing gum and control treatments could not be concealed from the patients, these were concealed from the authors.

\section{Interventions}

The upper dental arch of the patients had bonded edgewise brackets and simple tubes on the first molars with a slot size of $0.022 " \times 0.028$ ". The patients were randomly allocated to one of four groups: ibuprofen, acetaminophen, chewing gum, or control. Subsequently, the 0.014" nickel-titanium archwire (Morelli, Sorocaba$\mathrm{SP}$, Brazil) was placed and ligated using elastomeric ligatures. Patients allocated to the ibuprofen and acetaminophen groups were prescribed $400 \mathrm{mg}$ and $500 \mathrm{mg}$, respectively, of these analgesics immediately after initial archwire placement and every 6 hours for 1 week if the pain persisted. The chewing gum group was instructed to chew one tablet of chewing gum (Trident ${ }^{\circledR}$; Mondelez, Bauru, Brazil) for 5 minutes after initial archwire placement and for 5 minutes every 6 hours for the first week if pain occurred. The control group patients did not receive any pain relief but were not prohibited from using other analgesics. They were provided with instructions to inform the researchers if any other medication was used during the trial.

The patients were instructed to record their experiences of pain on a 100-mm visual analog scale (VAS) in a diary at the following time intervals: T1, 2 hours; T2, 24 hours; T3, 2 days; T4, 3 days; T5, 7 days; and T6, 21 days after initial archwire placement. At each time interval, the patients marked the VAS on two different parameters: the level of spontaneous pain with the mandible in the rest position (at rest) and the level of pain around the posterior teeth associated with maximum voluntary muscle contraction when biting down (while biting down). The patients received text messages on their cell phones alerting them to mark the VAS. They returned their diaries at T6 when the VAS records were measured using a millimeter ruler by a trained and blinded operator.

\section{Outcomes}

The primary outcome was the dependent variable pain. The VAS diaries were used to assess the experience of pain 2 and 24 hours as well as 2, 3, 7, and 21 days after initial archwire placement, both at rest and while biting down. The VASs consisted of 100-mm lines, on which $0 \mathrm{~mm}$ corresponded to no pain and $100 \mathrm{~mm}$ corresponded to unbearable pain.

\section{Blinding}

Blinding was possible for the ibuprofen and acetaminophen groups because the capsules were identical, and the labels on the containers did not identify the active ingredients. lbuprofen and acetaminophen containers were labeled as FORMULA 1 or FORMULA 2, respectively, and the identities of the contents were kept secret by the pharmacy company until the end of the trial.

\section{Sample size calculation}

Based on a variance of 16.37, the mean of the differences of $15 \mathrm{~mm}$, with a two-sided 5\% significance level, and power of 80\%, 19 patients per group were required for this study. Nevertheless, given an anticipated dropout rate of $20 \%, 24$ patients had to be included in each group. The variance of 16.37 was obtained in a pilot study conducted by the authors using a single time point of 24 hours after initial archwire placement. The mean difference of $15 \mathrm{~mm}$ was also obtained from a study in which the authors found that $15 \mathrm{~mm}$ on the VAS is the minimum clinically significant level of pain. ${ }^{17}$

\section{VAS measurement}

The VAS records were measured using a metal millimeter ruler by a trained operator blinded to the groups. Operator training consisted of the measurement of 20 VAS records, which were then remeasured 15 days later. The method's error was calculated using the Dahlberg formula, in which $\mathrm{D}$ is the difference between the first and second measurements, and $\mathrm{N}$ is the number of measurements performed. ${ }^{18}$ An acceptable error for the method (Sx) is < 3\%. ${ }^{19}$ The researcher that performed these measurements obtained an Sx of 1.38\%.

$$
\mathrm{Sx}=\frac{\Sigma \mathrm{D}^{2}}{2 \mathrm{~N}}
$$

\section{Statistical analysis}

The only change to the method after allocation concealment was regarding the statistical analysis. In addition to a per-protocol (PP) analysis using a completer analysis strategy, an intention-to-treat (ITT) analysis using a full random-sample strategy was performed to account for missing data. Missing data were obtained by multiple imputations in the ITT analysis. The statisti- 
cal analysis was performed using IBM SPSS Statistics for Windows/Macintosh, version 20.0 (IBM Corp., Armonk, NY, USA). The Kolmogorov-Smirnov and Shapiro-Wilk tests were used to determine the normality of data distribution of the dependent variable (pain). Since the data were non-normally distributed ( $p<0.0001$ ), nonparametric tests were used. The level of significance was set at $p<0.05$.

The chi-squared test was used to analyze the sex distribution among the groups, and the Kruskal-Wallis test was used to determine the distribution of patients according to age and upper dental arch discrepancy as well as the differences in recorded pain among the groups at each time point. The post-hoc Mann-Whitney $U$ test with Bonferroni correction was used to analyze data at each time point that showed significant differences on the Kruskal-Wallis test.

\section{RESULTS}

\section{Participation}

As of March 2018, enough patients had already been recruited for this study, considering an anticipated dropout rate of 20\%. The follow-up comprised the period from the clinic visit when the braces were bonded (baseline) until the first return visit (21 days after the baseline visit). In total, 106 patients aged 12-55 years old were randomized and allocated at a $1: 1: 1: 1$ ratio to the three



Figure 1. CONSORT flow diagram of the clinical trial.

VAS, visual analog scale; IT, intention-to-treat; PP, per-protocol.

Table 1. Demographic characteristics of the intervention and control groups analyzed on an intention-to-treat basis

\begin{tabular}{|c|c|c|c|c|}
\hline Characteristic & Ibuprofen $(n=27)$ & Acetaminophen $(n=26)$ & Chewing gum $(n=26)$ & Control $(n=27)$ \\
\hline \multicolumn{5}{|l|}{ Age (yr) } \\
\hline Minimum-Maximum & $12-45$ & $13-34$ & $12-55$ & $12-38$ \\
\hline Mean \pm SD & $19.25 \pm 8.00$ & $19.52 \pm 8.00$ & $22.42 \pm 13.00$ & $18.47 \pm 6.00$ \\
\hline $95 \% \mathrm{CI}$ & 15.51 to 22.99 & 15.57 to 23.49 & 16.59 to 28.26 & 15.56 to 21.40 \\
\hline \multicolumn{5}{|l|}{ Dental discrepancy } \\
\hline Mean \pm SD & $0.5 \pm 2.7$ & $-0.5 \pm 2.9$ & $-0.2 \pm 3.1$ & $1.6 \pm 3.8$ \\
\hline $95 \% \mathrm{CI}$ & -0.79 to 1.69 & -1.86 to +0.92 & -1.67 to +1.18 & -0.09 to 3.37 \\
\hline Ratio (male:female) & $15: 12$ & $12: 14$ & $12: 14$ & $13: 14$ \\
\hline
\end{tabular}

$\mathrm{SD}$, standard deviation; CI, confidence interval. 
intervention groups and the control group. Overall, 27, 26,26 , and 27 patients were allocated to the ibuprofen, acetaminophen, chewing gum, and control groups, respectively. Although all 106 patients were included in the ITT analysis, 7 each from the ibuprofen and acetaminophen groups, 5 from the chewing gum group, and 6 from the control group were excluded after monitoring based on the PP analysis. Patient enrollment is described in the CONSORT flow diagram (Figure 1).

\section{Baseline data}

Sample demographic characteristics (sex, age, dental discrepancy in the upper arch, and the male/female ratio) were similar among the four groups (Table 1). The chi-squared test showed that the three intervention groups and the control group had similar male/female ratios $(p=0.498)$. According to the Kruskal-Wallis test, the age and dental discrepancy of the upper arch in the patients were also similar ( $p=0.905$ and $p=0.423$, respectively). These baseline similarities were also observed in the PP analysis (male/female ratio, $p=0.496$; dental discrepancy, $p=0.442$; and age, $p=0.997$ ).

The Kruskal-Wallis test for the ITT analysis showed statistically significant differences at T2 (both at rest and while biting down), T3 (while biting down), and T4 (both at rest and while biting down) (all $p<0.05$ ) (Table 2). The PP analysis also showed statistically significant differences at T3 (while biting down) and T4 (both at rest and while biting down) (all $p<0.05$ ) (Table 3 ).

\section{ITT analysis}

The post-hoc Mann-Whitney $U$ test showed that there were not statistically significant differences between the groups at T2. The chewing gum group experienced lower pain levels at $\mathrm{T} 3$ at while biting down, than the ibuprofen $(p=0.04)$, acetaminophen ( $p=0.03)$, and control $(p=0.0006)$ groups. At T4, at rest, the chewing gum group showed significantly lower pain levels than the ibuprofen group $(p=0.001)$. While biting down, at $\mathrm{T} 4$, the chewing gum group also showed significantly lower pain levels than the acetaminophen $(p=0.02)$ and control $(p=0.0006)$ groups. Missing data were managed via multiple imputations. Two patients in the acetaminophen group and four in the control group used dipyrone, but their data were not excluded.

\section{PP analysis}

The PP analysis of patients who completed the study showed significant differences only between the chewing gum and control groups at T3 while biting down ( $p<$ $0.001)$ and at T4 both at rest $(p=0.030)$ and while biting down $(p=0.003)$.






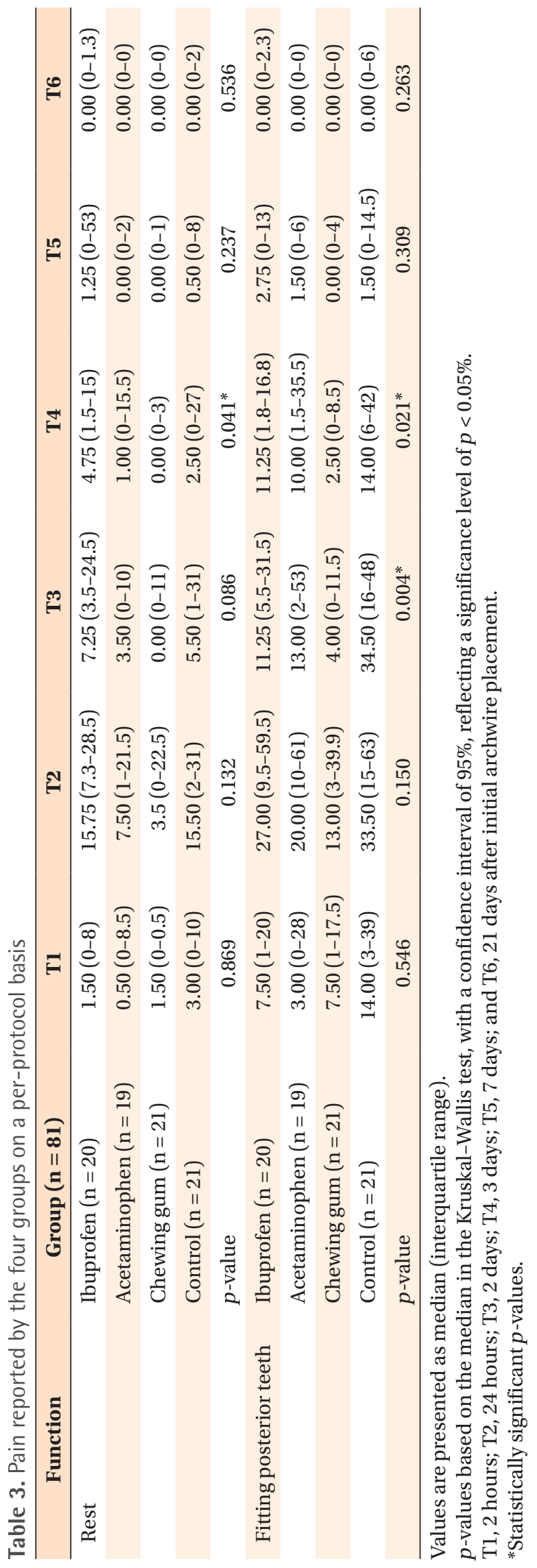

\section{Adverse events}

No adverse events were reported.

\section{DISCUSSION}

The statement that orthodontic pain peaks 24 hours after the application of force is flawed. No study performed prior to October 2015 had reported the actual time of force application and the next moment of pain evaluation. ${ }^{20}$ In this study, the time of each patient's initial archwire placement was registered and used as the initial point for marking the VAS.

Sandhu and Leckie $^{20}$ stated that pain would peak between 24 and 48 hours after applying the force, which indicates that the peak pain in the control group at the time of fitting the posterior teeth was not strictly at 24 hours, but rather at T3 (i.e., 2 days) in the present study (Tables 2 and 3). For sample size calculation, we assumed that 24 hours was when the orthodontic pain showed peak intensity based on the findings of previous reports ${ }^{13,21}$ and on the preliminary results of a pilot study.

The authors recommended acetaminophen at $750 \mathrm{mg}$. However, the Ethics Committee of the University where the study was performed disapproved of the dose once it was shown to potentially cause liver toxicity in children enrolled in the study. Therefore, the Committee suggested a dose reduction to $500 \mathrm{mg}$. Moreover, Kaur et al. ${ }^{14}$ evaluated the effectiveness of verbal behavior modification and acetaminophen on orthodontic pain; their findings suggested that the acetaminophen $(500 \mathrm{mg})$ group experienced more pain relief than the placebo and verbal behavior modification groups.

Farzanegan et al. ${ }^{5}$ instructed patients in the placebo control group to take vitamin capsules. In this protocol, patients in the ibuprofen group were blinded as the placebo and ibuprofen capsules were identical.

Otasevic et al. ${ }^{10}$ allocated 84 patients to a bite wafer group (BWG) or a reduced-mastication group (RMG). Patients in the BWG showed higher median pain than those in the RMG, probably because of the placebo instructions. Those in the RMG were told to avoid masticating hard food, while those in the BWG were told to chew the bite wafers to avoid pain. The patients in the BWG might have thought that pain was inevitable, resulting in higher reported levels of pain. Meanwhile, patients in the RMG could have interpreted the placebo instructions to mean that there would be no pain if they did not chew hard food.

Murdock et al. ${ }^{9}$ compared the effectiveness of bite wafers and over-the-counter (OTC) analgesics for relieving orthodontic pain. In contrast to the results of Otasevic et al., ${ }^{10}$ the bite wafer was not found to be inferior to OTC analgesics. However, Murdock et al. ${ }^{9}$ did not 
include a control group.

Benson et al. ${ }^{12}$ compared the effects of chewing gum versus no intervention on pain and the rate of appliance breakage. Although there was no placebo group, the results confirmed that the chewing gum group experienced lower pain intensity than the no intervention group.

Recently, Alqareer et al. ${ }^{7}$ reported the comparative efficacy of chewing gum and placebo in reducing orthodontic pain. Those in the placebo group were instructed to rinse their mouth for 30 seconds three times a day with a fluoride mouthwash, whereas those in the chewing gum group were instructed to use the same mouthwash and to chew gum for 5-10 minutes three times a day. Chewing gum did not relieve orthodontic pain when compared to the placebo. However, only the investigators in this study were blinded, and not the patients. Once the patients or their legal guardians provided written informed consent to participate in the study, it was impossible to blind the patients in either group.

In the present study, using a placebo to investigate the results from the chewing gum group would be complicated because the placebo would not allow successful blinding of patients in this group. Therefore, the present study featured a control group, in which the patients did not receive any intervention.

Moreover, in this study, no significant differences were observed between the groups at all time intervals in both the ITT and PP analyses. Nevertheless, the control group reported median pain values that tended to be higher than those of the acetaminophen and ibuprofen groups. This finding is consistent with the work of Monk et al., ${ }^{22}$ who showed that acetaminophen and NSAIDs like ibuprofen are effective at reducing orthodontic pain. Although our findings showed lower median scores by patients who were prescribed ibuprofen and acetaminophen when compared to control group, there were not statistically significant differences between ibuprofen and acetaminophen groups at time points of 2 and 24 hours after initial archwire placement.

Furthermore, the ibuprofen group showed a tendency toward higher median pain scores than did the acetaminophen group because drugs were administered postoperatively. According to Sandhu et al., ${ }^{23}$ ibuprofen is in the top 10 most effective interventions for orthodontic pain control only when it is administered both preoperatively and postoperatively.

Thus, ibuprofen's postoperative administration may have been insufficient to achieve analgesic effects like those of acetaminophen and chewing gum. Postoperative administration of NSAIDs is likely to be less effective because it is taken after the inflammatory cascade is triggered which means that inflammatory mediators are already accumulating in the periodontal ligament, sensi- tizing the nociceptors. ${ }^{24}$

Sandhu et al. ${ }^{23}$ also performed a meta-analysis that showed that analgesics and lasers could be used to effectively manage orthodontic pain. However, they affirmed that further research was required to improve the quality of evidence, especially for analgesic interventions. No chewing adjuncts were included in that study. The reason for the absence of RCTs on the effectiveness of chewing gum for orthodontic pain is probably due to the low quality of the RCTs. ${ }^{25}$

Bias in RCTs can only be minimized when data from all the patients are subjected to the ITT analysis. When patients or data are lost at random, the precision of the outcomes is affected. Therefore, the ITT analysis is recommended because it preserves the aims of randomization and maintains statistical power, on the basis that the initial matching of known and unknown factors and randomization are preserved. ${ }^{26}$

Furthermore, the results of the ITT analysis provide more information regarding the predicted effect of treatment in an average patient. In other words, the results include the impact of patients who do not comply with the treatment protocols, reflecting the "real-world" care situation. However, PP data are also useful for clinicians to inform their patients about the effects of treatments in an ideal setting. ${ }^{26}$

\section{Limitations of the study}

The VAS has limited reliability. During the interval between the baseline and follow-up appointments, the patients were monitored only via cell phone messages to ensure pain recording was performed. Despite this limitation, the response rate was reasonable, being approximately $74 \%, 73 \%, 81 \%$, and $78 \%$ for the ibuprofen, acetaminophen, chewing gum, and control groups, respectively.

The eligibility criterion of including patients with mild-to-moderate crowding that would allow the placement of a 0.014" nickel-titanium alloy initial archwire is empirical. This empiricism is justified because orthodontists are generally trained during their residency about appropriate archwire selection for each specific stage of the treatment. The degree of dental crowding of the patients' upper arches was measured via dental arch discrepancy analysis using a caliper and rule. In a hypothetical situation with a dental arch showing positive discrepancy because of diastema but also showing severe crowding in the incisors, a 0.014" nickel-titanium initial archwire may not be suitable.

The statistics regarding the number of patients who discontinued the interventions is currently lacking. It remains unclear whether the significant differences would have been observed if only the chewing gum group continued the intervention, and the pharmacological groups did not. 


\section{CONCLUSION}

Although the patients recorded that the peak orthodontic discomfort occurred 24 hours after initial archwire placement, no significant differences were observed among the groups at this time point. The use of a sugar-free chewing gum was effective for relieving orthodontic pain after initial archwire placement and can be a non-pharmacological alternative for managing orthodontic pain.

\section{CONFLICTS OF INTEREST}

No potential conflict of interest relevant to this article was reported.

\section{ACKNOWLEDGEMENTS}

We thank Prof. Ronir Raggio Luiz and Prof. Klaus Barreto for their assistance in the statistical analysis of the study data.

This study was part of the first author's Ph.D. program at the Rio de Janeiro State University. The first author received a scholarship from Coordenação de Aperfeiçoamento de Pessoal de Nivel Superior (Coordination of Superior Level Staff Improvement) CAPES-Brazil.

\section{REFERENCES}

1. Krishnan V. Orthodontic pain: from causes to management--a review. Eur J Orthod 2007;29:170-9.

2. Cheng $\mathrm{C}$, Xie T, Wang J. The efficacy of analgesics in controlling orthodontic pain: a systematic review and meta-analysis. BMC Oral Health 2020;20:259.

3. Bayani S, Rostami S, Ahrari F, Saeedipouya 1. A randomized clinical trial comparing the efficacy of bite wafer and low level laser therapy in reducing pain following initial arch wire placement. Laser Ther 2016;25:121-9.

4. Eslamipour F, Motamedian SR, Bagheri F. Ibuprofen and low-level laser therapy for pain control during fixed orthodontic therapy: a systematic review of randomized controlled trials and meta-analysis. J Contemp Dent Pract 2017;18:527-33.

5. Farzanegan F, Zebarjad SM, Alizadeh S, Ahrari F. Pain reduction after initial archwire placement in orthodontic patients: a randomized clinical trial. Am J Orthod Dentofacial Orthop 2012;141:169-73.

6. Al Shayea El. Comparative assessment between ibuprofen, chewing gum, and bite wafers in pain control following first archwire placement in orthodontic patients. J Contemp Dent Pract 2020;21:416-20.

7. Alqareer A, Alyahya A, Al-Anezi SA, AlAwadhi A, Al Qabandi S, Alyaseen M. Efficacy of chewing gum to reduce orthodontic pain compared to placebo: a blinded, parallel-group, preliminary clinical trial. J Oral Facial Pain Headache 2019;33:301-7.

8. Hwang JY, Tee CH, Huang AT, Taft L. Effectiveness of thera-bite wafers in reducing pain. J Clin Orthod 1994;28:291-2.

9. Murdock S, Phillips C, Khondker Z, Hershey HG. Treatment of pain after initial archwire placement: a noninferiority randomized clinical trial comparing over-the-counter analgesics and bite-wafer use. Am J Orthod Dentofacial Orthop 2010;137:316-23.

10. Otasevic M, Naini FB, Gill DS, Lee RT. Prospective randomized clinical trial comparing the effects of a masticatory bite wafer and avoidance of hard food on pain associated with initial orthodontic tooth movement. Am J Orthod Dentofacial Orthop 2006; 130:6.e9-15.

11. Proffit WR, Fields HW. The biologic basis of orthodontic therapy. In: Proffit WR, Fields HW, eds. Contemporary orthodontics. 3rd ed. St Louis: Mosby; 2000.

12. Benson PE, Razi RM, Al-Bloushi RJ. The effect of chewing gum on the impact, pain and breakages associated with fixed orthodontic appliances: a randomized clinical trial. Orthod Craniofac Res 2012; 15:178-87.

13. Ireland AJ, Ellis P, Jordan A, Bradley R, Ewings $P$, Atack NE, et al. Comparative assessment of chewing gum and ibuprofen in the management of orthodontic pain with fixed appliances: a pragmatic multicenter randomized controlled trial. Am J Orthod Dentofacial Orthop 2016;150:220-7.

14. Kaur H, Bansal N, Abraham R. A randomized, singleblind, placebo-controlled trial to evaluate the effectiveness of verbal behavior modification and acetaminophen on orthodontic pain. Angle Orthod 2019;89:617-23.

15. Hosseinzadeh Nik T, Shahsavari N, Ghadirian H, Ostad SN. Acetaminophen versus liquefied ibuprofen for control of pain during separation in orthodontic patients: a randomized triple blinded clinical trial. Acta Med Iran 2016;54:418-21.

16. Fleming PS, Strydom H, Katsaros C, MacDonald L, Curatolo M, Fudalej P, et al. Non-pharmacological interventions for alleviating pain during orthodontic treatment. Cochrane Database Syst Rev 2016;12: CD010263.

17. Angelopoulou MV, Vlachou V, Halazonetis DJ. Pharmacological management of pain during orthodontic treatment: a meta-analysis. Orthod Craniofac Res 2012;15:71-83.

18. Artun J, Grobéty D. Periodontal status of mandibular incisors after pronounced orthodontic advancement during adolescence: a follow-up evaluation. 
Am J Orthod Dentofacial Orthop 2001 119:2-10.

19. de Souza Galvão MC, Sato JR, Coelho EC. Dahlberg formula: a novel approach for its evaluation. Dental Press J Orthod 2012;17:115-24.

20. Sandhu SS, Leckie G. Orthodontic pain trajectories in adolescents: between-subject and within-subject variability in pain perception. Am J Orthod Dentofacial Orthop 2016;149:491-500.e4.

21. Patel S, McGorray SP, Yezierski R, Fillingim R, Logan $\mathrm{H}$, Wheeler TT. Effects of analgesics on orthodontic pain. Am J Orthod Dentofacial Orthop 2011; 139:e53-8.

22. Monk AB, Harrison JE, Worthington HV, Teague A. Pharmacological interventions for pain relief during orthodontic treatment. Cochrane Database Syst Rev 2017;11:CD003976.

23. Sandhu SS, Cheema MS, Khehra HS. Comparative effectiveness of pharmacologic and nonpharmacologic interventions for orthodontic pain relief at peak pain intensity: a Bayesian network meta-analysis. Am J Orthod Dentofacial Orthop 2016;150:13-32.

24. Kim TW, Ryu YK. Experimental study of the effects of non-steroidal anti-inflammatory agents used in denial clinic on orthodontic tooth movement in rats. Korean J Orthod 1992;22:579-87.

25. Pandis N, Tu YK, Fleming PS, Polychronopoulou A. Randomized and nonrandomized studies: complementary or competing? Am J Orthod Dentofacial Orthop 2014;146:633-40.

26. Barretto Dos Santos Lopes Batista K, Thiruvenkatachari B, O’Brien K. Intention-to-treat analysis: are we managing dropouts and missing data properly in research on orthodontic treatment? A systematic review. Am J Orthod Dentofacial Orthop 2019; 155:19-27.e3. 